BNL-114205-2017-JA

\title{
A coherent discrete variable representation method on a sphere
}

\author{
H.-G. Yu
}

Submitted to J. Chem. Phys.

August 2017

Chemistry Department

Brookhaven National Laboratory

\author{
U.S. Department of Energy \\ USDOE Office of Science (SC), \\ Basic Energy Sciences (BES) (SC-22)
}

\footnotetext{
Notice: This manuscript has been authored by employees of Brookhaven Science Associates, LLC under Contract No. DE- SC0012704 with the U.S. Department of Energy. The publisher by accepting the manuscript for publication acknowledges that the United States Government retains a non-exclusive, paid-up, irrevocable, world-wide license to publish or reproduce the published form of this manuscript, or allow others to do so, for United States Government purposes.
} 


\section{DISCLAIMER}

This report was prepared as an account of work sponsored by an agency of the United States Government. Neither the United States Government nor any agency thereof, nor any of their employees, nor any of their contractors, subcontractors, or their employees, makes any warranty, express or implied, or assumes any legal liability or responsibility for the accuracy, completeness, or any third party's use or the results of such use of any information, apparatus, product, or process disclosed, or represents that its use would not infringe privately owned rights. Reference herein to any specific commercial product, process, or service by trade name, trademark, manufacturer, or otherwise, does not necessarily constitute or imply its endorsement, recommendation, or favoring by the United States Government or any agency thereof or its contractors or subcontractors. The views and opinions of authors expressed herein do not necessarily state or reflect those of the United States Government or any agency thereof. 


\title{
A coherent discrete variable representation method on a sphere
}

\author{
Hua-Gen $\mathrm{Yu}^{1}$ \\ Division of Chemistry, Department of Energy and Photon Sciences, \\ Brookhaven National Laboratory, Upton, NY 11973-5000, USA
}

(August 16, 2017)

\begin{abstract}
The coherent discrete variable representation (ZDVR) has been extended for constructing a multidimensional potential-optimized DVR basis on a sphere. In order to deal with the non-constant Jacobian in spherical angles, two direct product primitive basis methods are proposed so that the original ZDVR technique can be properly implemented. The method has been demonstrated by computing the lowest states of a two dimensional (2D) vibrational model. Results show that the extended ZDVR method gives accurate eigenvalues and exponential convergence with increasing ZDVR basis size.
\end{abstract}

\footnotetext{
${ }^{1}$ E-mail: hgy@bnl.gov; Fax:+1-631-344 5815
} 


\section{Introduction}

The discrete variable representation (DVR) technique ${ }^{1-8}$ has played an important role in chemical physics. ${ }^{9-12}$ In DVR, a function of coordinates red alone (such as the potential energy surface) is diagonal, which avoids any numerical or analytical integration. The DVR method is not only simple but also highly accurate in terms of the Gauss quadrature theory $^{1-3}$ of classical polynomials. Nowadays, one often uses even more efficient DVRs, the potential-optimized (PO) DVRs. ${ }^{4,5}$ Since the PODVRs are determined using a onedimensional (1D) reduced Hamiltonian with a problem-adapted reference potential, only a few PODVRs are required for an accurate solution of a variety of physical systems. It shows a quasi-Gauss quadrature accuracy. ${ }^{4,5}$ For high dimensional problems, a direct product (1D) DVR method is usually used. The resulting basis is truncatable.

Although the 1D PODVR method is very powerful, red there has been little progress in developing accurate multidimensional PODVR basis functions. A few ideas or framework were suggested using semiclassical and phase space concepts. ${ }^{13-15}$ In order to determine multi-dimensional PODVRs, one requires simultaneous diagonalization of coordinate matrices for the relevant degrees-of-freedom. Since two different coordinate matrices do not in general commute, this simultaneous diagonalization red is mathematically impossible. Instead, an approximate simultaneous diagonalization (SD) DVR method has been proposed by Dawes and Carrington, ${ }^{16}$ and Manthe. ${ }^{17}$ The SD-DVR method can provide moderately accurate results. A different method for a 2D PODVR has been developed in this laboratory $^{18}$ incorporating a pair of coordinates $x$ and $y$ into a single complex variable, $z=x+i y$. Since the $z$ coordinate matrix can be diagonalized using the c-norm condition, ${ }^{19}$ this approach, named the coherent discrete variable representation and abbreviated as ZDVR, is an effective means of constructing 2D PODVRs. The ZDVR method shows exponential convergence with basis size, and gives accurate eigenstates. It is a very attractive approach for reducing the size of a basis used for accurate variational solutions to problems in polyatomic dynamics, particularly if it can be extended to the $(\theta, \phi)$ anglular components of 
multiple polyspherical coordinates. ${ }^{20,21}$ This extension to spherical coordinates is the point of this paper.

red It has been a long-standing problem to get an efficient standard DVR method on a sphere (described by two angles $\theta$ and $\phi$ ), which is ubiquitous in quantum Hamiltonian in polyspherical coordinates. ${ }^{20,21}$ Currently, one usually employs the cubature approach, including the Lebedev quadrature scheme $\mathrm{e}^{22-24}$ and the pseudospectral transform techniques. $^{25-28}$ In those methods, the grid basis size is larger than that of finite basis representation (FBR). As a result, the DVRs are not orthonormal so that they are not truncatable. Consequently, it will become very expensive and demanding of memory for high dimensional problems. In order to circumvent this difficulty, one often uses a crude $1 \mathrm{D}$ direct product DVR method that may lead to the singularity problem at $\sin \theta=0$. Recently, Schiffel and Manthe ${ }^{29}$ proposed a specially treated cotangent DVR method to alleviate the singularity problem. Until now, however, there has been no good multidimensional PODVR method available for polyspherical angles.

In this work, we report a ZDVR method on a sphere. Since the original ZDVR method ${ }^{18}$ is developed based on a constant Jacobian, it is not straightforward to apply it to the spherical system owing to the Jacobian $J=\sin \theta$. Actually, it is a constrained 3D problem with a unit radial coordinate $r=1$. The simple use of $z=\cos \theta+i \cos \phi$ would not work properly. Two approaches, explicit and implicit, are proposed to cope with the nonconstant Jacobian. As the ZDVR theory has been previously well documented, ${ }^{18}$ here, we will focus on only the construction of coordinate and kinetic energy operators, especially in the polar angle $\theta$.

\section{ZDVR on a sphere}

In order to illustrate and verify the proposed ZDVR method, we will use the following 2D Hamiltonian,

$$
\hat{H}(\theta, \phi ; \lambda)=\frac{g}{2}\left(\hat{t}_{k}+\frac{1}{\sin ^{2} \theta} \frac{\partial^{2}}{\partial \phi^{2}}\right)+V(\theta, \phi ; \lambda)
$$


with the kinetic energy operator

$$
\hat{t}_{k}=-\frac{1}{\sin \theta} \frac{\partial}{\partial \theta} \sin \theta \frac{\partial}{\partial \theta}
$$

and the $2 \mathrm{D}$ potential energy surface

$$
V(\theta, \phi ; \lambda)=\cot ^{2} \theta+\frac{1}{2}(1+\cos \phi)^{2}+\frac{\lambda}{2}(1+\cos \phi)\left(\cot ^{2} \theta-\frac{1}{12}(1+\cos \phi)^{2}\right) .
$$

red It is a 2D Hénon-Heiles-like potential with two harmonic oscillators plus a perturbative anharmonicity and coupling, as is often used for numerical testing. ${ }^{40}$ The coupling strength parameter is taken as $\lambda=0.111803$ as used in Ref. [30]. In Eq. (1) the prefactor $g$ is set at 0.025 so that the eigenvalues of the Hamiltonian are similar to common molecular vibrational energies in $\mathrm{eV}$. In addition, we will also discuss the expression of the anti-Hermitian operator ${ }^{31}$

$$
\hat{p}_{\theta}=\frac{\partial}{\partial \theta}+\frac{1}{2} \cot \theta
$$

that is required for further application to high dimensional systems, although it is not used in this work.

Following our previous work, ${ }^{18}$ the ZDVRs are obtained by diagonalizing a complex symmetric matrix $\mathbf{Z}=\mathbf{X}+i \mathbf{Y}$ of the coordinate $z=x+i y$,

$$
\mathrm{ZQ}=\mathrm{Q} \Lambda_{z}
$$

in a set of $2 \mathrm{D}$ basis functions $\left\{\mid \psi_{m}>, m=1, \cdots, N_{\text {ZDVR }}\right\}$ (defined in Eq. (11) below) with the c-norm condition. ${ }^{19}$ A c-normalized state is represented by a round ket as $\mid \cdots$ ) in contrast to a normal state $\mid \cdots>$. Here, we use the coordinate variables $y=\cos \phi$ and $x=\cot \theta$ or $\cos \theta$. The choice of $x$ will be discussed below. The diagonal matrix $\Lambda_{z}=\Lambda_{x}+i \Lambda_{y}$ defines the ZDVR pivots at the diagonal elements $z_{\alpha}=x_{\alpha}+i y_{\alpha}$. Both $\Lambda_{x}$ and $\Lambda_{y}$ are real matrices. According to transformation theory, ${ }^{1}$ the ZDVR basis functions $\left.\mid z_{\alpha}\right)$ are obtained by the transformation matrix $\mathbf{Q}$ as

$$
\left.\left.\left.\left\{\mid z_{1}\right), \mid z_{2}\right), \cdots, \mid z_{N_{\mathrm{ZDVR}}}\right)\right\}=\left\{\left|\psi_{1}>,\right| \psi_{2}>, \cdots, \mid \psi_{N_{\mathrm{ZDVR}}}>\right\} \mathbf{Q}
$$


with

$$
\begin{gathered}
\left(z_{\alpha} \mid z_{\beta}\right)=\int \psi_{\alpha}(z) \psi_{\beta}(z) d z=\delta_{\alpha \beta}, \\
\left.\sum_{\alpha=1}^{N_{\text {ZDVR }}} \mid z_{\alpha}\right)\left(z_{\alpha} \mid=\mathbf{I} .\right.
\end{gathered}
$$

By using the $\mathbf{Q Q}^{T}=\mathbf{I}$ identity, we have the reverse basis transformation

$$
\left.\left.\left.\left\{\left|\psi_{1}>,\right| \psi_{2}>, \cdots, \mid \psi_{N_{\mathrm{ZDVR}}}>\right\}=\left\{\mid z_{1}\right), \mid z_{2}\right), \cdots, \mid z_{N_{\mathrm{ZDVR}}}\right)\right\} \mathbf{Q}^{T}
$$

In addition, all ZDVR properties hold for their complex conjugates, for instance,

$$
\mathbf{Z}^{*} \mathbf{Q}^{*}=\mathbf{Q}^{*} \Lambda_{z}^{*}
$$

As in traditional DVR theory ${ }^{3}$ for real coordinates, any coordinate function in ZDVR is local.

The 2D basis functions $\mid \psi_{m}>$ are formed by the lowest orthonormal eigenstates of a reference Hamiltonian $\hat{H}^{0}(x, y)$, i.e.

$$
\hat{H}^{0}(x, y)\left|\psi_{m}>=E_{m}^{0}\right| \psi_{m}>
$$

where the reference Hamiltonian $\hat{H}^{0}$ is taken as the Hamiltonian in Eq. (1) with $\lambda=0$. The eigenvalue problem of $\hat{H}^{0}$ is solved in a direct product basis $\left\{\left|\varphi_{x j}>\right| \varphi_{y k}>\right\}$ of primitive orthonormal basis functions in each degree of freedom. They are labeled as $\left\{\left|\varphi_{x j}\right\rangle\right.$, $\left.j=1, \cdots, N_{\theta}\right\}$ in the $x$ coordinate and $\left\{\mid \varphi_{y k}>, k=1, \cdots, N_{\phi}\right\}$ in $y$. An eigenstate is expressed as

$$
\left|\psi_{m}>=\sum_{j, k} C_{j k}^{m}\right| \varphi_{x j}>\mid \varphi_{y k}>.
$$

Then the coordinate matrices are given by

$$
\begin{aligned}
& \mathbf{X}_{n m}=<\psi_{n}|x| \psi_{m}>=\sum_{j^{\prime}, j, k} C_{j^{\prime} k}^{n *} C_{j k}^{m} \mathbf{x}_{j^{\prime} j}, \\
& \mathbf{Y}_{n m}=<\psi_{n}|y| \psi_{m}>=\sum_{j, k^{\prime}, k} C_{j k^{\prime}}^{n *} C_{j k}^{m} \mathbf{y}_{k^{\prime} k},
\end{aligned}
$$

with the one-dimensional coordinate matrices

$$
\mathbf{x}_{j^{\prime} j}=<\varphi_{x j^{\prime}}|x| \varphi_{x j}>\text { and } \mathbf{y}_{k^{\prime} k}=<\varphi_{y k^{\prime}}|y| \varphi_{y k}>
$$


In this work, the primitive basis functions in $\phi$ are defined by $(2 M+1)\left(=N_{\phi}\right)$ orthonormal Fourier functions, ${ }^{32}$ namely,

$$
\mid \varphi_{y k}>=\frac{1}{\sqrt{2 \pi}} e^{i(k-M-1) \phi}, k=1, \cdots, N_{\phi}
$$

which gives a coordinate matrix as

$$
\mathbf{y}_{k^{\prime} k}=\frac{1}{2}\left(\delta_{k^{\prime}, k+1}+\delta_{k^{\prime}+1, k}+\delta_{k^{\prime}, 1} \delta_{k, N_{\phi}}+\delta_{k, 1} \delta_{k^{\prime}, N_{\phi}}\right)
$$

The primitive DVR pivots and collocation matrix are defined by its eigenvalues and eigenvectors. In particular, in the Fourier basis set, both the first and second derivatives are diagonal, i.e.

$$
\frac{\partial}{\partial \phi}\left|\varphi_{y k}>=(k-M-1) i\right| \varphi_{y k}>\text { and } \frac{\partial^{2}}{\partial \phi^{2}}\left|\varphi_{y k}>=-(k-M-1)^{2}\right| \varphi_{y k}>.
$$

For other $\phi$-related kinetic operators, there also exist analytical expressions. The reader can refer elsewhere. ${ }^{31,32}$ Here one should beware that Fourier functions are not classical orthogonal polynomials. ${ }^{32}$ Its coordinate matrix is not tri-diagonal. red The $\cos \phi$ coordinate has a dual-valued representation within $[0,2 \pi]$. The $\phi$ range is determined by using the sign of parity of sine function. It can be calculated by the diagonal matrix element of $\mathbf{Q}_{r}^{T} \mathbf{p} \mathbf{Q}_{r}$, where $\mathbf{Q}_{r}$ and $\mathbf{p}$ are the real part of $\mathbf{Q}$ and a diagonal parity matrix of primitive basis functions, respectively. In addition, it is also good to directly use $\phi$ for the calculation of DVR pivots, in which the periodic boundary condition should be properly implemented.

On the other hand, the primitive basis functions in $\theta$ will be constructed from a set of raw normalized functions $\left\{\mid \xi_{\tilde{j}}>, \tilde{j}=-2, \cdots, N_{\theta}-3\right\}$, red but such functions are not fully orthogonal (see below for detail). The overlap matrix is given by $\tilde{\mathbf{S}}$ with the element $\tilde{\mathbf{S}}_{\tilde{j}^{\prime} \tilde{j}}=<\xi_{\tilde{j}^{\prime}} \mid \xi_{\tilde{j}}>$. Accordingly, the matrix representations of the coordinate $x$ and operator $\hat{O}\left(=\hat{t}_{k}\right.$ and $\left.\hat{p}_{\theta}\right)$ are given by $\tilde{\mathbf{x}}$ and $\tilde{\mathbf{O}}$, respectively, in the raw basis set. Their matrices in the primitive basis $\mid \varphi_{x j}>$ can be calculated through diagonalizing the overlap matrix,

$$
\tilde{\mathbf{S}} \mathbf{U}=\mathbf{U} \Lambda_{s} .
$$


Then we have

$$
\begin{aligned}
\mathbf{x} & =\boldsymbol{\Lambda}_{s}^{-1 / 2} \mathbf{U}^{T} \tilde{\mathbf{x}} \mathbf{U} \boldsymbol{\Lambda}_{s}^{-1 / 2} \\
\mathbf{O} & =\boldsymbol{\Lambda}_{s}^{-1 / 2} \mathbf{U}^{T} \tilde{\mathbf{O}} \mathbf{U} \boldsymbol{\Lambda}_{s}^{-1 / 2}
\end{aligned}
$$

Similarly, by diagonalizing the coordinate matrix $\mathbf{x}$, one can obtain the primitive DVRs and transformation matrix between DVR and FBR in the $\theta$ coordinate. In the next subsection, we will discuss how to calculate the $\tilde{\mathbf{S}}, \tilde{\mathbf{x}}$, and $\tilde{\mathbf{O}}$ matrices in the $\left\{\mid \xi_{\tilde{j}}>\right\}$ basis set.

Once one has obtained the ZDVR basis set, the eigenstates of the complete Hamiltonian in Eq. (1) can be solved by using a direct diagonalization method (or an iterative diagonalization method for high dimensional applications). The performance of the ZDVR method can be investigated in detail.

\subsection{Explicit LP-cotDVR approach}

The ZDVR method described above requires two important properties: an appropriate treatment of the non-constant Jacobian and a good set of raw basis functions. In order to impose the Jacobian $J=\sin \theta$, here, we use an explicit approach. That is, the coordinate $x$ is selected to be $\cot \theta$ instead of the traditional $\cos \theta$. In such a manner, the $x=\cot \theta$ coordinate behaves like $\cos \theta$ with a constant Jacobian after the cancellation of the $\sin \theta$ factor. In addition, the raw basis functions are given by

$$
\left\{\mid \xi_{\tilde{j}}>, \tilde{j}=-2,-1,0, \cdots, N_{\theta}-3\right\}=\left\{|21>,| 11>,|00>,| 10>, \cdots, \mid\left(N_{\theta}-3\right) 0>\right\}
$$

where $\mid l m>=\Theta_{l}^{m}(\theta)$ are the normalized associated Legendre polynomials including the phase factor. Thus, this method is denoted as an explicit LP-cotDVR (Legendre polynomialcotangent DVR) approach. The two functions $\mid 21>$ and $\mid 11>$ are added to correctly describe the singularity at $\sin \theta=0$. They are used to compensate for the flaw of the Legendre polynomials $\mid l 0>$. A similar technique was used by Schiffel and Manthe ${ }^{29}$ for Chebyshev polynomials. Their numerical results showed that this technique works very well. 
The basis set is normalized but the two groups $\mid j 1>$ and $\mid l 0>$ are not orthogonal with each other. The overlap matrix elements are written as

$$
\tilde{\mathbf{S}}_{\tilde{j} \tilde{l}}=\left\{\begin{array}{ll}
1 & \text { if } \tilde{j}=\tilde{l} \\
\frac{1}{2} \sqrt{\frac{(2 j+1)(2 l+1)}{j(j+1)}} I(j, 1 ; l, 0) & \text { if }\left(\left|\xi_{\tilde{j}}>=\right| j 1>\text { and }\left|\xi_{\tilde{l}}>=\right| l 0>\right) \\
& \text { or }\left(\left|\xi_{\tilde{j}}>=\right| l 0>\text { and }\left|\xi_{\tilde{l}}>=\right| j 1>\right) \\
0 & \text { otherwise }
\end{array},\right.
$$

where $I\left(l_{1}, m_{1} ; l_{2}, m_{2}\right)$ is the overlap integral of associated Legendre polynomials $\left(P_{l}^{m}(\theta)\right)$ that can be evaluated analytically. ${ }^{33}$ In this basis set, the kinetic energy operator $\left(\hat{t}_{k}\right)$ matrix is obtained as

$$
\left[\tilde{\mathbf{t}}_{k}\right]_{\tilde{j} \tilde{l}}=\left\{\begin{array}{ll}
\tilde{j}^{2}-\frac{1}{2} & \text { if } \tilde{j}=\tilde{l} \leq-1 \\
\tilde{l}(\tilde{l}+1) & \text { if } \tilde{j}=\tilde{l} \geq 0 \\
l(l+1) \tilde{\mathbf{S}}_{\tilde{j} \tilde{l}} & \text { if }\left(\left|\xi_{\tilde{j}}>=\right| j 1>\text { and }\left|\xi_{\tilde{l}}>=\right| l 0>\right) \\
& \text { or }\left(\left|\xi_{\tilde{j}}>=\right| l 0>\text { and }\left|\xi_{\tilde{l}}>=\right| j 1>\right) \\
0 & \text { otherwise }
\end{array} .\right.
$$

Before determining the coordinate $x=\cot \theta$ and operator $\hat{p}_{\theta}$ matrices, let us first derive the matrix $\tilde{\mathbf{D}}$ of the partial derivative operator $\partial / \partial \theta$. Its matrix elements are obtained as

$$
\tilde{\mathbf{D}}_{\tilde{j} \tilde{l}}=\left\{\begin{array}{ll}
\frac{1}{4} \sqrt{\frac{\left(2 j^{\prime}+1\right)(2 j+1)}{j^{\prime}\left(j^{\prime}+1\right) j(j+1)}} I\left(j^{\prime}, 1 ; j, 2\right)-\frac{1}{4} \sqrt{\frac{\left(2 j^{\prime}+1\right)(2 j+1) j(j+1)}{j^{\prime}\left(j^{\prime}+1\right)}} I\left(j^{\prime}, 1 ; j, 0\right) \\
\text { if }\left|\xi_{\tilde{j}}>=\right| j^{\prime} 1>\text { and }\left|\xi_{\tilde{l}}>=\right| j 1> \\
\frac{1}{4} \sqrt{\frac{(2 l+1)(2 j+1)}{j(j+1)}} I(l, 0 ; j, 2)-\frac{1}{2} \sqrt{j(j+1)} \delta_{l j} & \text { if }\left|\xi_{\tilde{j}}>=\right| l 0>\text { and }\left|\xi_{\tilde{l}}>=\right| j 1> \\
\frac{1}{2} \sqrt{\frac{\left(2 j^{\prime}+1\right)(2 l+1)}{j^{\prime}\left(j^{\prime}+1\right)}} I\left(j^{\prime}, 1 ; l, 1\right) & \text { if }\left|\xi_{\tilde{j}}>=\right| j^{\prime} 1>\text { and }\left|\xi_{\tilde{l}}>=\right| l 0> \\
\frac{1}{2} \sqrt{\left(2 l^{\prime}+1\right)(2 l+1)} I\left(l^{\prime}, 0 ; l, 1\right) & \text { if }\left|\xi_{\tilde{j}}>=\right| l^{\prime} 0>\text { and }\left|\xi_{\tilde{l}}>=\right| l 0>
\end{array} .\right.
$$

With the help of the relationship, ${ }^{34}$

$$
\frac{\partial^{\dagger}}{\partial \theta}=-\frac{\partial}{\partial \theta}-\cot \theta
$$

the $x$ and $\hat{p}_{\theta}$ matrices can be easily computed as

$$
\tilde{\mathbf{x}}=-\left(\tilde{\mathbf{D}}^{T}+\tilde{\mathbf{D}}\right) \text { and } \tilde{\mathbf{p}}_{\theta}=-\frac{1}{2}\left(\tilde{\mathbf{D}}^{T}-\tilde{\mathbf{D}}\right)
$$

where the $\tilde{\mathbf{x}}$ matrix is symmetric while the $\tilde{\mathbf{p}}_{\theta}$ matrix is anti-Hermitian as expected. It is very helpful that all relevant matrices can be computed analytically. 


\subsection{Implicit sw-cosDVR approach}

Alternatively, in this subsection, we will describe an implicit method to handle the Jacobian problem by using a weight function $w(\theta)=1 / \sin \theta$. In other words, we will use a set of raw basis functions that are normalized with respect to the weight function, i.e

$$
<\xi_{\tilde{j}}|w| \xi_{\tilde{j}}>=\int \xi_{\tilde{j}}^{*} \sin \theta \xi_{\tilde{j}} w d \theta=1
$$

where the Jacobian $J=\sin \theta$ is imposed by $w(\theta)$. In such a way, we can still use the normal $\cos \theta$ as the coordinate $x=\cos \theta$ for the DVR calculations. Obviously, Chebyshev polynomials are ideal basis functions. Following the same arguments discussed above, the selected raw basis functions are

$$
\left\{\mid \xi_{\tilde{j}}>, \tilde{j}=-2,-1,0, \cdots, N_{\theta}-3\right\}=\left\{\left|-\frac{1}{2}>,\right| \frac{1}{2}>,|0>,| 1>, \cdots, \mid\left(N_{\theta}-3\right)>\right\}
$$

where $\mid l>$ are the normalized Chebyshev polynomials,

$$
\left\{\begin{array}{rl}
\mid-\frac{1}{2}> & =\sqrt{\frac{2}{\pi}} \sin \frac{1}{2} \theta \\
\mid \frac{1}{2}> & =\sqrt{\frac{2}{\pi}} \cos \frac{1}{2} \theta \\
\mid n> & =\sqrt{\frac{2}{\left(1+\delta_{0 n}\right) \pi}} \cos n \theta, \quad n=0,1, \cdots
\end{array} .\right.
$$

Thus, this method is called as an implicit sw-cosDVR (1/sine weighted cosine-DVR) method. Similarly, the two functions $\mid \pm \frac{1}{2}>$ are used for the boundary conditions at $\sin \theta=0$.

In this basis set, the overlap matrix is very sparse. The non-zero elements are given by

$$
\left\{\begin{array}{ll}
\tilde{\mathbf{S}}_{\tilde{j j}}=1, & \tilde{j}=-2,-1, \cdots, N_{\theta}-3, \\
\tilde{\mathbf{S}}_{-2,-1}=\tilde{\mathbf{S}}_{-1,-2}=\frac{2}{\pi}, & \\
\tilde{\mathbf{S}}_{-2, n}=\tilde{\mathbf{S}}_{n,-2}=-\frac{4}{\sqrt{1+\delta_{0 n}}\left(4 n^{2}-1\right) \pi}, & n \geq 0 \\
\tilde{\mathbf{S}}_{-1, n}=\tilde{\mathbf{S}}_{n,-1}=(-1)^{n} \tilde{\mathbf{S}}_{-2, n}, & n \geq 0
\end{array} .\right.
$$

For the coordinate $(x=\cos \theta)$ matrix, the non-zero elements are written as

$$
\begin{cases}\tilde{\mathbf{x}}_{-2,-2}=-\frac{1}{2} & \\ \tilde{\mathbf{x}}_{-1,-1}=\frac{1}{2} & \\ \tilde{\mathbf{x}}_{-1, n}=\tilde{\mathbf{x}}_{n,-1}=\frac{1}{2 \sqrt{1+\delta_{0 n}}}\left(\tilde{\mathbf{S}}_{-1, n+1}+\sqrt{1+\delta_{0, n-1}} \tilde{\mathbf{S}}_{-1,|n-1|}\right), & n \geq 0 \\ \tilde{\mathbf{x}}_{-2, n}=\tilde{\mathbf{x}}_{n,-2}=-(-1)^{n} \tilde{\mathbf{x}}_{-1, n}, & n \geq 0 \\ \tilde{\mathbf{x}}_{0,1}=\tilde{\mathbf{x}}_{1,0}=\frac{\sqrt{2}}{2}, & n \geq 1 \\ \tilde{\mathbf{x}}_{n, n+1}=\tilde{\mathbf{x}}_{n+1, n}=\frac{1}{2}, & \end{cases}
$$


Furthermore, the anti-Hermitian $\hat{p}_{\theta}$ matrix is again calculated by

$$
\tilde{\mathbf{p}}_{\theta}=-\frac{1}{2}\left(\tilde{\mathbf{D}}^{T}-\tilde{\mathbf{D}}\right)
$$

where $\tilde{\mathbf{D}}$ is the matrix of partial derivative $\partial / \partial \theta$. It is obtained as

$$
\begin{cases}\tilde{\mathbf{D}}_{-2,-2}=\frac{1}{\pi} \\ \tilde{\mathbf{D}}_{-1,-2}=\frac{1}{2}, & \\ \tilde{\mathbf{D}}_{n,-2}=\frac{1}{2} \tilde{\mathbf{S}}_{-1, n}, & n \geq 0 \\ \tilde{\mathbf{D}}_{-2,-1}=-\frac{1}{2}, & \\ \tilde{\mathbf{D}}_{-1,-1}=-\frac{1}{\pi}, & n \geq 0 \\ \tilde{\mathbf{D}}_{n,-1}=-\frac{1}{2} \mathbf{S}_{-2, n}, & n \geq 0 \\ \tilde{\mathbf{D}}_{-2, n}=\frac{(-1)^{n} 8 n^{2}}{\left(4 n^{2}-1\right) \pi}, & n \geq 0 \\ \tilde{\mathbf{D}}_{-1, n}=-\frac{8 n^{2}}{\left(4 n^{2}-1\right) \pi}, & n \geq 0 \\ \tilde{\mathbf{D}}_{0, n}=-\frac{\left[1-(-1)^{n}\right] \sqrt{2}}{\pi}, & n \geq 0 \text { and } m \geq 1 \\ \tilde{\mathbf{D}}_{m, n}=-\frac{\left[1-(-1)^{n+m}\right] n}{\pi}\left[\frac{1}{n+m}+\frac{1-\delta_{n m}}{n-m}\right], & \end{cases}
$$

However, we are unable to derive an analytical expression for the kinetic energy operator $\hat{t}_{k}$. Instead, its matrix is calculated in term of the relationship

$$
\hat{t}_{k}=\left(\frac{\partial}{\partial \theta}-\frac{1}{2} \cot \theta\right)^{T}\left(\frac{\partial}{\partial \theta}-\frac{1}{2} \cot \theta\right)
$$

in the DVR basis set.

\section{Numerical results}

In this section, we numerically test and discuss the performance of the ZDVR method for studying the eigenstates of the 2D model Hamiltonian described above. The exact eigenvalues are computed by using the pseudospectral transform method ${ }^{25,26}$ in a set of non-direct product spherical harmonics $Y_{l m}(\theta, \phi)(m=-l, \cdots, l)$ with $l_{\max }=36$. It gives a total of 1369 FBR functions that converge the lowest 12 eigenvalues better than $10^{-8}$. In the ZDVR calculations, 73 primitive Fourier functions were used in $\phi$ and 37 raw basis functions were employed in $\theta$ for both the explicit and implicit approaches.

Table 1 lists a comparison of the ZDVR results with the exact values for the lowest 12 eigenstates. The errors are given by the differences between the ZDVR value and the exact value for each level. It shows that those levels can be well converged with a rather 
small basis set. Highly accurate results are determined at $N_{\text {ZDVR }}=65$ for both the LPcotDVR and sw-cosDVR methods, demonstrating the advantage of the multidimensional PODVR technique. Furthermore, Fig. 1 shows a logarithmic plot of the root-mean-square (rms) errors as a function of the ZDVR basis size. The explicit LP-cotDVR ZDVR method converges the states slightly faster than the implicit sw-cosDVR ZDVR approach. Importantly, both methods show a nearly exponential convergence. Therefore, the new ZDVR method is very successful for the spherical problem.

The 45 ZDVR quadrature points are shown in Fig. 2 for a better understanding of ZDVRs. They are calculated with a direct product basis set of $N_{\theta}=25$ and $N_{\phi}=49$. The 1D DVRs are also shown in each degree-of-freedom. red First, if we diagonalize the $z$ coordinate matrix for a complete set of $N_{\theta} \times N_{\phi}$ ZDVRs without any reference potential, we obtain a set of direct product pivots for the ZDVR quadrature points. This is an important property. Furthermore, with the 2D quadratic reference potential, the 45 ZDVR points well scatter around the local potential minimum as expected. Actually, they are a set of optimal basis set with respect to the phase space of the reference Hamiltonian. ${ }^{8,13-15}$

\section{Conclusion}

In summary, we have developed a coherent discrete variable representation (ZDVR) on a sphere. It has overcome the difficulty of the non-constant Jacobian. Results show that the novel ZDVR method is very accurate and efficient for the variational study of systems in polyspherical coordinates. Both the explicit and implict ZDVR methods show a great performance. Between them, the explicit LP-cotDVR ZDVR method gives a slightly better convergence speed. Although the method is demonstrated in a $2 \mathrm{D}$ case, it is very straightforward to apply the ZDVR method for studying high dimensional problems via a direct product ZDVR technique, as has been described in our previous work. ${ }^{18}$

Finally, it is worthwhile to mention that the LP-cotDVR and sw-cosDVR methods can also be applied for constructing 1D (PO)DVR in polar angle for a general system. They (including $\hat{p}_{\theta}$ ) have been tested by calculating the rovibrational states of $\mathrm{C}_{2} \mathrm{H}_{3}$ and $\mathrm{CH}_{2} \mathrm{OH}$ 
radicals in full dimensionality. Results obtained are very good.

\section{Acknowledgments}

The author would like to thank Dr. Gregory E. Hall for his helpful comments and reading the manuscript. This work was performed at Brookhaven National Laboratory under Contract No. DE-SC0012704 with the U.S. Department of Energy, Office of Science, and supported by its Division of Chemical Sciences, Geosciences, and Biosciences within the Office of Basic Energy Sciences. It also used the resource at the National Energy Research Scientific Computing Center (NERSC) under Contract No. DE-AC02-05CH11231. 


\section{References}

[1] D.O. Harris, G.G. Engerholm, and W.D. Gwinn, J. Chem. Phys. 43, 1515 (1965).

[2] A.S. Dickinson and P.R. Certain, J. Chem. Phys. 49, 4209 (1968).

[3] J.C. Light, I.P. Hamilton, and J.V. Lill, J. Chem. Phys. 82, 1400 (1985).

[4] J. Echave and D.C. Clary, Chem. Phys. Lett. 190, 225 (1992).

[5] H. Wei and T. Carrington, Jr., J. Chem. Phys. 97, 3029 (1992).

[6] J.C. Light and T. Carrington Jr., Adv. Chem. Phys. 114, 263 (2000).

[7] V. Szalay, J. Chem. Phys. 125, 154115 (2006).

[8] V. Szalay and P. Adam, J. Chem. Phys. 137, 064118 (2012).

[9] T. Carrington, Jr., J. Chem. Phys. 146, 120902 (2017).

[10] D.H. Zhang and H. Guo, Ann. Rev. Phys. Chem. 67, 135 (2016).

[11] A.G. Csazar, C. Fabri, T. Szidarovsky, E. Matyus, T. Furtenbacher, and G. Czako, Phys. Chem. Chem. Phys. 14, 1085 (2012).

[12] J.C. Tremblay, G. Fuechsel, and P. Saalfrank, Phys. Rev. B 86, 045438 (2012).

[13] R.G. Littlejohn, M. Cargo, T. Carrington Jr., K.A. Mitchell, and B. Poirier, J. Chem. Phys. 116, 8691 (2002).

[14] B. Poirier and J.C. Light, J. Chem. Phys. 111, 4869 (1999).

[15] B. Yang, W. Chen, and B. Poirier, J. Chem. Phys. 135, 094306 (2011).

[16] R. Dawes and T. Carrington Jr., J. Chem. Phys. 121, 726 (2004).

[17] U. Manthe, J. Chem. Phys. 130, 054109 (2009).

[18] H.-G. Yu, J. Chem. Phys. 122, 164107 (2005). 
[19] N. Moiseyev, Non-Hermitian Quantum Mechanics (Cambridge University Press, Cambridge, 2011).

[20] F. Gatti and C. Iung, Phys. Rep. 484, 1 (2009).

[21] G. Nyman and H.-G. Yu, Int. Rev. Phys. Chem. 32, 39 (2013).

[22] V.I. Lebedev, Zh. Vychisl. Mat. Mat. Fiz. 15, 48 (1975).

[23] V.I. Lebedev, Zh. Vychisl. Mat. Mat. Fiz. 16, 293 (1976).

[24] D.J. Haxton, J. Phys. B 40, 4443 (2007).

[25] G.C. Corey, J.W. Tromp, and D. Lemoine, in NATO ARW Proceedings on Grid Methods in Atomic and Molecular Quantum Calculation (Kluwer Academic, Dordrecht, The Netherland, 1993).

[26] C. Cerjan ed., Numerical Grid Methods and Their Application to Schrödinger's Equation (Klumer Academic Publishers, USA, 1993).

[27] R.A. Friesner, J. Chem. Phys. 85, 1462 (1986).

[28] H.-G. Yu, Chem. Phys. Lett. 281, 312 (1997).

[29] G. Schiffel and U. Manthe, Chem. Phys. 374, 118 (2010).

[30] O. Vendrell and H.-D. Meyer, J. Chem. Phys. 134, 044135 (2011).

[31] H.-G. Yu, J. Chem. Phys. 117, 2030 (2002).

[32] J.T. Muckerman, Chem. Phys. Lett. 173, 200 (1990).

[33] B.R. Wong, J. Phys. A 31, 1101 (1998).

[34] B. Poirier, Chem. Phys. 308, 305 (2005). 
Table 1: Errors of eigenvalues calculated with the ZDVR method (via the LP-cotDVR (Explicit) and the sw-cosDVR (Implicit) approaches) relative to the exact values for the lowest 12 states of the 2D Hénon-Heiles-like potential with the ZDVR basis sizes of 25, 45, and 65. The root-mean-square (rms) errors are listed in the last row.

\begin{tabular}{|c|c|c|c|c|c|c|}
\hline Exact & Explicit $^{a}$ & Implicit & Explicit & Implicit & Explicit & Implicit \\
\hline 1369 & \multicolumn{2}{|c|}{25} & \multicolumn{2}{|c|}{45} & \multicolumn{2}{|c|}{65} \\
\hline 0.14044409 & $3.9(-7)$ & $8.2(-7)$ & $2.6(-7)$ & $3.4(-7)$ & $2.5(-7)$ & $3.2(-7)$ \\
\hline 0.21297078 & $5.7(-6)$ & $6.1(-6)$ & $2.9(-7)$ & $5.9(-7)$ & $2.6(-7)$ & $3.3(-7)$ \\
\hline 0.30671195 & $7.1(-7)$ & $2.9(-6)$ & $2.6(-7)$ & $7.2(-7)$ & $2.8(-7)$ & $3.4(-7)$ \\
\hline 0.39152259 & $-7.5(-7)$ & $1.5(-6)$ & $3.1(-7)$ & $9.7(-7)$ & $3.1(-7)$ & $3.9(-7)$ \\
\hline 0.41061766 & $-5.2(-4)$ & $-2.8(-4)$ & $3.7(-7)$ & $-1.9(-6)$ & $2.8(-7)$ & $3.5(-7)$ \\
\hline 0.46960009 & $-6.0(-4)$ & $-6.9(-4)$ & $-6.1(-6)$ & $3.3(-6)$ & $9.0(-8)$ & $4.1(-7)$ \\
\hline 0.52167201 & $3.1(-5)$ & $2.8(-4)$ & $5.0(-7)$ & $-1.4(-6)$ & $2.2(-7)$ & $3.2(-7)$ \\
\hline 0.56971778 & $-1.5(-4)$ & $8.8(-5)$ & $5.9(-7)$ & $9.4(-7)$ & $2.1(-7)$ & $4.7(-7)$ \\
\hline 0.63778169 & $-7.4(-5)$ & $3.0(-2)$ & $2.4(-6)$ & $9.2(-6)$ & $2.6(-7)$ & $3.2(-7)$ \\
\hline 0.66757318 & $1.2(-4)$ & $8.4(-2)$ & $5.1(-7)$ & $3.6(-6)$ & $1.5(-7)$ & $2.5(-7)$ \\
\hline 0.68024847 & $7.7(-2)$ & $1.3(-1)$ & $-8.4(-6)$ & $2.3(-5)$ & $2.6(-7)$ & $3.6(-7)$ \\
\hline 0.75111453 & $4.2(-2)$ & $1.1(-1)$ & $9.1(-6)$ & $4.4(-5)$ & $5.0(-7)$ & $2.5(-7)$ \\
\hline $\mathrm{rms}$ & $2.5(-2)$ & $5.5(-2)$ & $4.1(-6)$ & $1.5(-5)$ & $2.7(-7)$ & $3.5(-7)$ \\
\hline
\end{tabular}

${ }^{a}$ The number in parentheses is the power of ten. 
Figure 1: A logarithmic plot of the root-mean-square (rms) errors of the lowest 12 eigenvalues calculated with the ZDVR method via either the explicit LP-cotDVR (open circles) or the implicit sw-cosDVR (open triangles) schemes as a function of ZDVR basis size $\left(N_{\text {ZDVR }}\right)$ for the 2D Hénon-Heiles-like potential.

Figure 2: The 45 ZDVR quadrature points (filled circles) projected on the 2D contour surface of the Hénon-Heiles potential. The contour potential values are 0.05, 0.1, 0.2, 0.5, 1.0 and 2.0 in the order of the smallest circle to the largest one. The 1D DVRs in each coordinate are also shown in open triangles at the edges. The upper and lower panels are the explicit LP-cotDVR and implicit sw-cosDVR ZDVRs, respectively. 


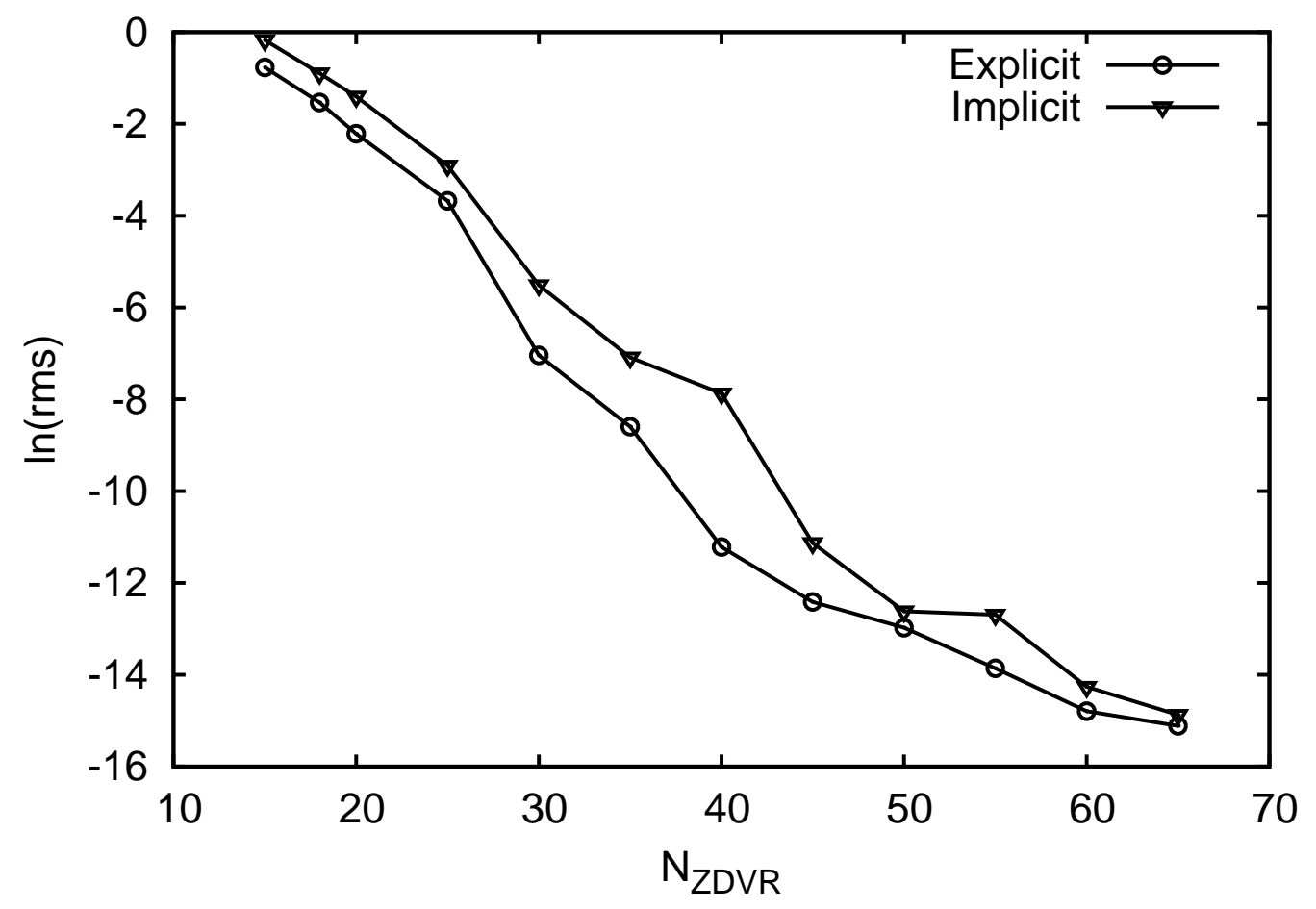

Fig. 1 

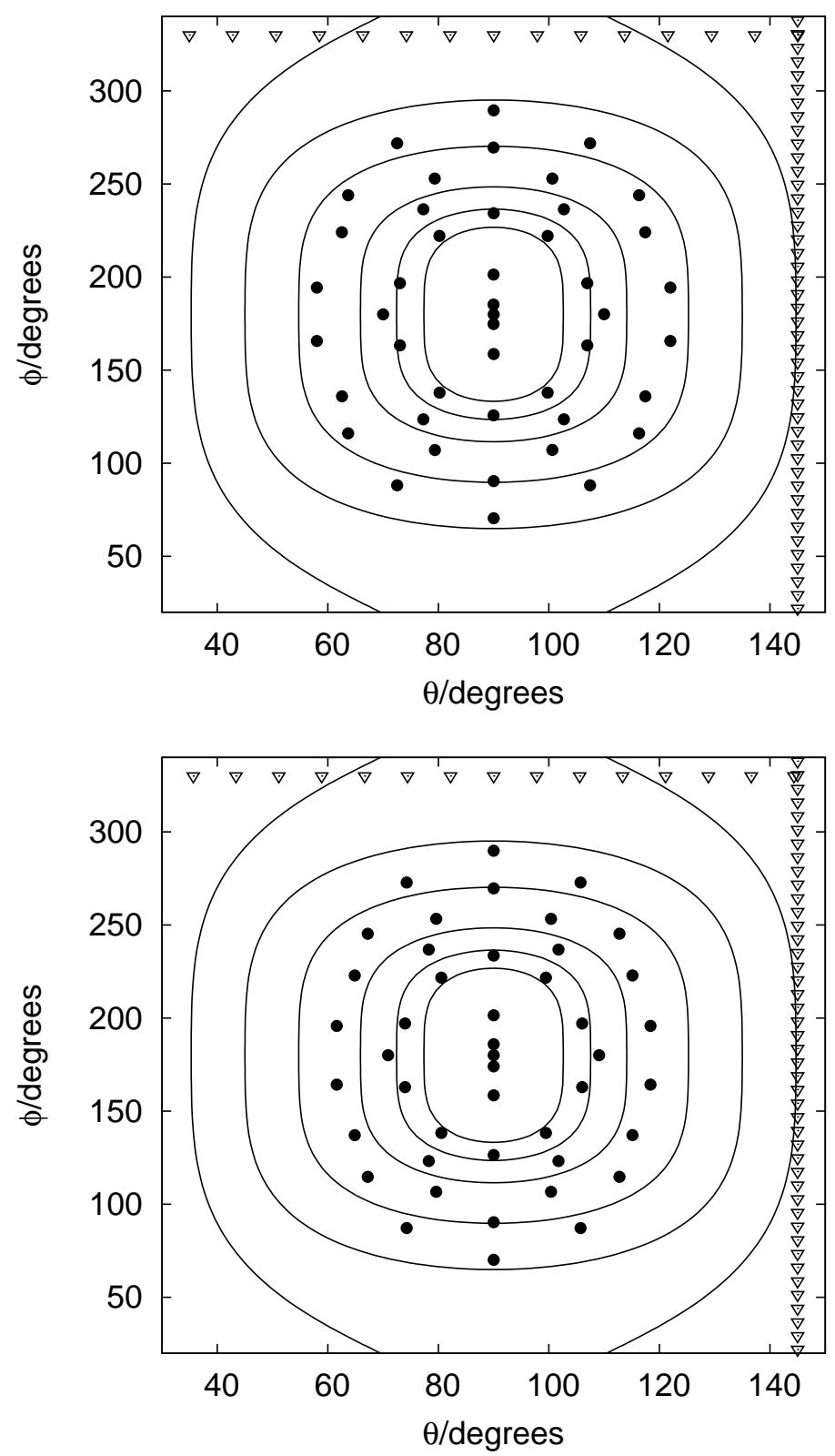

Fig. 2 